SAE paper 2007-01-0825 Copyright (C) 2007 SAE International. This paper is posted on this site with permission from SAE International, and is for viewing only. Further use or distribution of this paper is not permitted without permission from SAE. 


\title{
Robust Active Roll Controller Design for Vehicles Considering Variable Speed and Actuator Delay
}

\author{
Haiping Du \\ Nong Zhang \\ Guangming Dong \\ University of Technology, Sydney
}

Copyright $\odot 2007$ SAE International

\begin{abstract}
A robust controller design method for vehicle roll control with variable speed and actuator delay is presented. Based on a three-degree-of-freedom (3DOF) yaw-roll model, the $\mathrm{H}_{\infty}$ performance from the steering input to the vehicle body roll angle is considered. The design approach is formulated in terms of the feasibility of delay-dependent matrix inequalities. By combining the random search of genetic algorithms (GAs) and the efficient solution of linear matrix inequalities (LMIs), the state feedback controllers can be obtained. The approach is validated by simulations showing that the designed controllers can achieve good performance in roll control.
\end{abstract}

\section{INTRODUCTION}

Rollover crashes of vehicles, especially heavy goods vehicles, sport utility vehicles (SUV), etc., are worldwide public safety issues. Preventing rollover crashes is becoming crucial for safe highway operation. Rollover prevention can be achieved by employing rollover warning and anti-rollover systems [1]. Since most existing rollover warning systems are based on signal threshold techniques and the warning actions are turned on only when the vehicle roll angle or the lateral acceleration exceeds a pre-selected value, they are usually conservative and do not predict impending rollover danger in the future. Hence, active roll control $(A R C)$ is necessary to assist drivers in rollover prevention. Rollover normally occurs when the vehicle is unable to provide a stabilizing net restoring moment in response to an overturning moment. The objective of active rollover prevention is to provide the vehicle with an ability to resist overturning moments generated during cornering. To date, several schemes of active control strategies have been proposed in the literature for vehicle rollover reduction, e.g., active steering, active suspension, active stabilizer, and differential braking, etc. In $[2,3,4]$, special emphasis has been placed on the use of active roll control systems in heavy road vehicles to improve roll stability and reduce the likelihood of rollover accidents.

Many vehicles, such as commercial vehicles, regularly operate in highly variable environments. Parameter variations resulting from loading patterns and driving conditions will influence the vehicle dynamics. Therefore, it is required that the designed ARC system should be robust stable to the variations of the vehicle parameters within reasonable bounds. For a heavy vehicle model with time-variable vehicle forward velocity, a customary linear interpolation method is used in [3] to obtain the interpolated control law between the fixed speed controllers. A gain-scheduled controller and a linear parameter-varying (LPV) controller are developed in [5] and [6], respectively, to deal with the variations of vehicle dynamics assuming real-time measurement of varying forward speed is available. Regarding the load variation, it was verified in [2] that the designed linear quadratic regulator (LQR) controller could guarantee the stability of the controlled vehicle in the presence of model uncertainties within some indicated bounds. Furthermore, the parameter variations are considered in [5] as model uncertainties, and robust $\mathrm{H}_{\infty}$ control theory is applied there to design the robust controller.

For an engineering system, unavoidable time delays always appear in the controlled channel. The sum of time delays is due to on-line data acquisition, filtering, processing of data, calculating control forces and transmitting the control force signals from the computer to the actuator, particularly in the digital controllers as they carry out the calculations associated with complex sophisticated control laws, and in the actuator hardware, such as large hydraulic actuators where delays are taken by the actuators to build up required control force. For an ARC system, hydraulic actuators are used to 
provide the active control forces, where the time delay effect when the control forces are applied to the vehicle is becoming an important issue. Though the delay time may be short, it can nevertheless limit the control performance or even cause instability of the system when the delay appears in the feedback loop. However, in most research works on ARC systems, time delay of the actuator has not yet been considered.

Following the above discussion, this paper is concerned with the robust controller design for a three-degree-offreedom (3DOF) yaw-roll vehicle model with time delay in the control input. The focal point of the paper is on developing an algorithm to design the state feedback control law such that the closed-loop system is asymptotically stable with a prescribed level of disturbance attenuation subject to a variation of vehicle forward speed and input delay within allowable bounds. Sufficient conditions for designing such a controller are given in terms of a matrix inequalities based on the recent result in [7]. Combined with the random search of genetic algorithms (GAs), the feasible solution is obtained by solving a finite number of linear matrix inequalities (LMIs). Simulation results show that despite the time delay in control input and the variation of vehicle forward speed, the designed state feedback controller can achieve good roll control performance. The approach can be applied to design the partial-state feedback controller without further modification.

\section{VEHICLE DYNAMICS MODEL}

In order to develop the active controller, it is not desirable to use a nonlinear complex vehicle model due to sampling time and implementation of the control system. In this paper, a three degree-of-freedom (3DOF) yaw-roll model is used for the design of the controller.

Vehicle parameters of a 1997 Jeep Cherokee published in [1] were used to construct the 3DOF model based on Lagrangian dynamics. The 3DOF yaw-roll model is shown in Figure 1.

Including the direct roll moment control as one of the control inputs, the equation of motion in matrix form for the 3DOF yaw-roll model is written as

$$
E \dot{x}(t)=A_{0} x(t)+B_{0} u(t)+B_{w 0} w(t),
$$

where $x(t)=\left[\begin{array}{llll}v(t) & r(t) & p(t) & \phi(t)\end{array}\right]^{T} ; v(t), r(t), p(t)$, and $\phi(t)$ are lateral velocity, yaw rate, roll rate and roll angle of sprung mass, respectively; $u(t)$ is an active roll moment; $w(t)=\delta_{\text {tire }}(t)$ is steering disturbance;

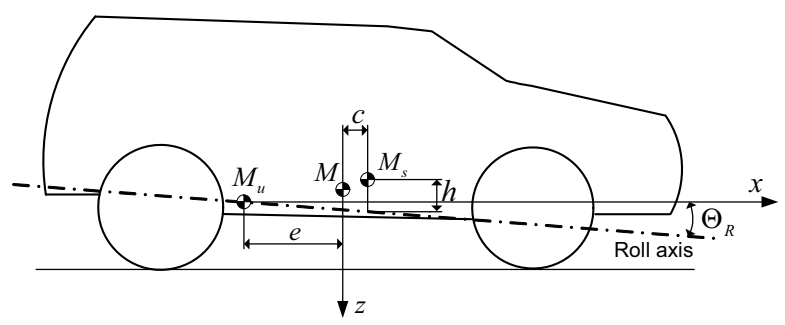

(a) Side view

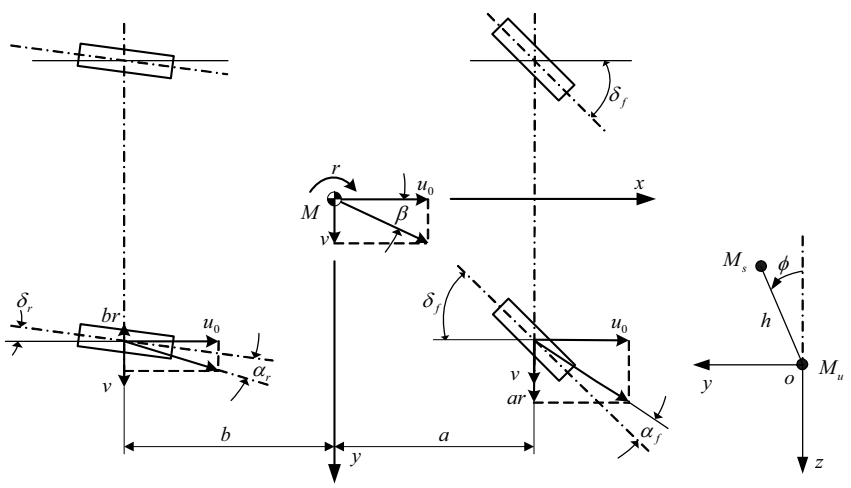

(b) Top view

(c) Front view

Figure 1. 3 DOF yaw-roll model

$$
\begin{aligned}
E & =\left[\begin{array}{cccc}
M & 0 & M_{s} h & 0 \\
0 & I_{z} & I_{x z} & 0 \\
M h & I_{x z} & I_{x} & 0 \\
0 & 0 & 0 & 1
\end{array}\right], B_{w 0}=\left[\begin{array}{c}
Y_{\delta} \\
N_{\delta} \\
0 \\
0
\end{array}\right], \\
A_{0} & =-\left[\begin{array}{cccc}
-Y_{\beta} / u_{0} & M u_{0}-Y_{r} & 0 & -Y_{\phi} \\
-N_{\beta} / u_{0} & -N_{r} & 0 & -N_{\phi} \\
0 & M h u_{0} & -L_{p} & -L_{\phi} \\
0 & 0 & -1 & 0
\end{array}\right], B_{0}=\left[\begin{array}{l}
0 \\
0 \\
1 \\
0
\end{array}\right] .
\end{aligned}
$$

Detailed descriptions of other entries in the above equation are referred to in [1] and are given in the Appendix.

\section{CONTROLLER DESIGN}

The time delay of the actuator, especially the hydraulic actuator, can not be avoided in engineering. With consideration of the actuator time delay, the 3DOF yawroll model is expressed with a state space equation as

$$
\dot{x}(t)=A x(t)+B u(t-\tau)+B_{w} w(t)
$$

where $A=E^{-1} A_{0}, B=E^{-1} B_{0}, B_{w}=E^{-1} B_{w 0}$, and $\tau$ is the time delay of the control input, which is realized by hydraulic actuators, satisfying $0 \leq \tau \leq \bar{\tau}$, where $\bar{\tau}$ is the maximum time delay allowed. For an actual vehicle, it is expected that the vehicle can operate in a highly variable environment. Hence, parameter variations resulting from loading patterns and driving conditions will 
be unavoidable and these variations will considerably influence vehicle dynamics. When the varied parameter, vehicle forward velocity $u_{0}$, is considered in the model, the vehicle model becomes an uncertain system which is dependent on the time-varying parameter $u_{0}$. Actually, it is noticed from (2) that only the $A$ matrix is related to $u_{0}$ and $1 / u_{0}$ which can be defined as two parameters $\eta_{1}$ and $\eta_{2}$, respectively. And the vertices $\xi_{i}(i=1, \ldots, 4)$ are defined as $\xi_{1}=\left[\begin{array}{ll}\eta_{1 \mathrm{~min}} & \eta_{2 \mathrm{~min}}\end{array}\right]$, $\xi_{2}=\left[\begin{array}{ll}\eta_{1 \max } & \eta_{2 \min }\end{array}\right] \quad, \quad \xi_{3}=\left[\begin{array}{ll}\eta_{1 \min } & \eta_{2 \max }\end{array}\right] \quad, \quad$ and $\xi_{4}=\left[\begin{array}{ll}\eta_{1 \max } & \eta_{2 \max }\end{array}\right]$. If we define $\alpha_{1}=x y$, $\alpha_{2}=(1-x) y, \alpha_{3}=x(1-y)$, and $\alpha_{4}=(1-x)(1-y)$, where $x=\frac{\eta_{1 \max }-\eta_{1}}{\eta_{1 \max }-\eta_{1 \min }}$, and $y=\frac{\eta_{2 \max }-\eta_{2}}{\eta_{2 \max }-\eta_{2 \min }}$, then matrix $A$ can be expressed as $A=\sum_{i=1}^{4} \alpha_{i} A_{i}$, where $A_{i}$ is the value of $A$ at the vertex $\xi_{i}$. Therefore, the uncertain system (2) belongs to a polytopic set described by four vertices.

In this paper, we are interested in designing a parameter independent controller $u(t)=K x(t)$ for system (2) such that the closed-loop system is stable with performance $\left\|T_{z w}\right\|_{\infty}<\gamma$ achieved for any $\gamma>0$ and $0 \leq \tau \leq \bar{\tau}$, where $T_{z w}$ is the closed-loop transfer function from the disturbance $w(t)$ to the control output $z(t)$.

\section{MATRIX INEQUALITIES EXPRESSION}

Combining the controller $u(t)=K x(t)$ with equation (2) gives

$$
\begin{aligned}
& \dot{x}(t)=A x(t)+B K x(t-\tau)+B_{w} w(t) \\
& z(t)=C x(t) \\
& x(t)=\phi(t), \quad \forall t \in[-\tau, 0]
\end{aligned}
$$

where $C=\left[\begin{array}{llll}0 & 0 & 0 & 1\end{array}\right]$ to define the control output as the roll angle, and $\phi(t)$ is the initial condition. The following theorem gives the sufficient condition for system (3) to be asymptotically stable for any time delay satisfying $0 \leq \tau \leq \bar{\tau}$ with performance $\left\|T_{z w}\right\|_{\infty}<\gamma$.

Theorem 1: Consider system (3), if there exist constant matrices $P>0, Q>0, Z>0, H>0$, and a matrix $V$ satisfying

$$
\left[\begin{array}{ccccc}
\Pi & P B K-V & P B_{w} & \bar{\tau} A_{i}^{T} Z & C^{T} \\
* & -Q & 0 & \bar{\tau}(B K)^{T} Z & 0 \\
* & * & -\gamma^{2} I & \bar{\tau} B_{w}^{T} Z & 0 \\
* & * & * & \bar{\tau} Z & 0 \\
* & * & * & * & -I
\end{array}\right]<0, \forall i=1, . ., 4
$$

$\left[\begin{array}{ll}H & V \\ * & Z\end{array}\right] \geq 0$

where $\Pi=P A_{i}+A_{i}^{T} P+\bar{\tau} H+V+V^{T}+Q$, then the closed-loop system is robustly stable with disturbance attenuation $\gamma$ for any time delay $0 \leq \tau \leq \bar{\tau}$.

The proof of Theorem 1, which is omitted here for brevity, is fully based on the Theorem 1 presented in [7]. Notice that Theorem 1 provides a delay-dependent condition for stability and $\mathrm{H}_{\infty}$ performance analysis, respectively.

\section{COMPUTATIONAL ALGORITHM}

Genetic algorithm is a stochastic search algorithm based on simulation of the human trial-and-error procedure using the Darwinian principle of "survival of the fittest". One important property of GAs is that they optimize an objective function globally through stochastic transitions of points from one generation to another, thus preventing being trapped at local solutions.

The matrix inequality conditions in Theorem 1 are not LMIs in terms of the decision matrix variables. In the following, an algorithm which combines the random search capability of GAs with the solvability of LMls will be proposed. Specifically, we will find a desirable controller $K$ by solving the following maximization problem:

$$
\max _{K_{i} \in \mathbf{R}} \quad \bar{\tau} \quad \text { subject to LMI }
$$

In this problem, the GA is used to randomly generate an initial matrix $K_{i}$ which changes thereafter within the evolution procedure according to objective (5). If (5) is feasible for an evolved $K_{i}$ which has the maximum $\bar{\tau}$, then this $K_{i}$ satisfies the specifications and thus constitutes a solution to the design problem. Note that the matrix inequalities (4) are LMls once the control gain matrix $K_{i}$ is known, and the LMls can be solved efficiently by using the efficient convex optimization algorithm. Furthermore, the partial-state (static output) feedback controller can be designed as well by defining an appropriate control matrix.

When the search space for the unknown parameters is known a priori, the search efficiency of the GA for the 
optimal parameters can be high. However, when the search space cannot be determined in a relatively small region before the searching process begins, the searching for the optimal parameters will be timeconsuming and sometimes there is no guarantee to obtain the optimal parameters. On the other hand, if the initial parameter search region is fixed throughout the searching process, it may prevent the searching of the global optimum point(s). Therefore, it is necessary to choose a small initial search space, and to expand the search space dynamically in the GA algorithm.

One parameter search space expansion scheme, in which the upper and lower bound for each parameter is expanded during a checking phase introduced periodically over generations of iterations, is adopted here. For instance, in this scheme, if the $l$-th parameter $k_{l}$ is positive and $\bar{k}_{l} / 2 \leq k_{l} \leq \bar{k}_{l}$ is satisfied within the checking phase, where $\bar{k}_{l}$ is the upper bound of $k_{l}$, the $\bar{k}_{l}$ value will be set to $\varepsilon \bar{k}_{l}$, where $\varepsilon>1$ is a preset expansion factor and the selection of $\varepsilon$ depends on the size of the initial search space. The strategy used for expanding the lower bound of parameter $k_{l}$ is similar. When any parameter search interval has been expanded, a new population will be generated for the expanded search space and the costs of its members are evaluated. A new initial population is then formed by selecting those better members among the evolved population and the newly generated population, which will be used for the expanded space subsequently.

Combining the above mentioned strategies with the standard GA, the final algorithm for the controller design using a binary-coded GA can be realized. Since the standard genetic algorithms can be found in most related textbooks, and our algorithm used in this work is similar to that described in reference [8], the details of the algorithm are omitted here for brevity. Only an outline is given as follows:

Step 1: Randomly generate an initial population of $N_{p}$ individuals containing controller candidates using binary number chromosomes. The chromosome for the controller gain can be generated in the form of $\underbrace{11011 \cdots}_{k_{1}}|\underbrace{00101 \cdots}_{k_{2}}| \cdots \cdots$, where each controller gain coefficient $k_{l}$ is coded as a binary string.

Step 2: Evaluate the fitness of each individual according to the objective in (5). Firstly, use the randomly generated parameter set to construct every controller gain matrix $K_{i}, i=1,2, \ldots, N_{p}$. For every $K_{i}$, use the bisection method to search for the maximum delay $\tau_{i}$ such that with a delay $\tau_{i}$ and $K_{i}$, LMIs in (4) are feasible. Take every delay $\tau_{i}$ as the objective value corresponding to $K_{i}$ and associate every $K_{i}$ with a suitable fitness value according to a rank-based fitness assignment approach, and then go to Step 4. If for a $K_{i}$ there is no feasible delay which can be found such that LMls in (4) are feasible, the objective value corresponding to $K_{i}$ will be assigned a large value in order to reduce its opportunity to survive in the next generation.

Step 3: Based on the fitness obtained in Step 2, the offspring is chosen for the next crossover and mutation steps by using the tournament selection approach. The tournament selection is one of many methods of selection in GAs which runs a "tournament" among a few individuals chosen at random from the population and selects the winner (the one with the best fitness).

Step 4: Perform recombination (crossover) with probability $p_{c}$ and mutation operation with a small mutation probability $p_{m}$ to the current population to generate new individuals in the search space. The crossover operation generates new points in the search space by exchanging genetic materials between "good" chromosomes. In the uniform crossover scheme, which is used in this paper, the newly selected chromosomes in the new population are randomly paired together. In each pair of chromosomes, the bits are probabilistically and independently swapped at each bit position with crossover probability to produce the new pair of chromosomes. The bit mutation operation that simply flips each bit (changing a 1 to a 0 and vice versa) in the population of chromosomes with the mutation probability is used in this paper.

Step 5: An elitist reinsertion strategy is used to guarantee that the best chromosome in the population always survives and is retained in the next generation. The elitist reinsertion strategy is realized by replacing the worst parents (those with the lowest fitness rating) with the new children.

Step 6: Execute the search space expansion scheme mentioned above when the generation number is a multiple of the checking period.

Steps 3 to 6 correspond to one generation. The evolution process will repeat for $N_{g}$ generations or will be ended when the search process converges with a given accuracy.

\section{SIMULATION RESULTS}

In this section, we will apply the proposed approach to design the robust controller based on the 3DOF yaw-roll model described in Section 2. The parameters of the 3DOF 1997 Jeep Cherokee yaw-roll model selected for this study are listed in the Appendix. The basic GA parameters used in this paper are as follows: 
$N_{p}=80, p_{c}=0.8, p_{m}=0.01$, and $N_{g}=300$.

Assuming that all the state variables defined in equation (4) are measurements available for feedback, we can design a full-state feedback controller in this case. We assume that the forward velocity can vary from $36 \mathrm{~km} / \mathrm{h}$ to $180 \mathrm{~km} / \mathrm{h}(10 \mathrm{~m} / \mathrm{s}$ to $50 \mathrm{~m} / \mathrm{s})$. Using the present algorithm, we obtain the full-state feedback controller gain matrix as:

$$
K=10^{3} \times\left[\begin{array}{llll}
-1.1967 & 0.7217 & -1.1969 & -1.1505
\end{array}\right] .
$$

Actually, using the presented approach, this controller is feasible for the maximum time-delay of $\bar{\tau}=25 \mathrm{~ms}$. This means that the controller can stabilize the system (2) with the $\mathrm{H}_{\infty}$ performance index $\gamma=10$ for any time-delay satisfying $0<\bar{\tau}<25 \mathrm{~ms}$.

To evaluate the performance of the designed controller, the theoretical steady state performance of the full-state feedback controller is compared to a passive suspension in Figure 2, where the roll angle vs lateral acceleration is plotted under the operation condition of constant vehicle forward speed $90 \mathrm{~km} / \mathrm{h}$, and input delay $\tau=0$. It can be seen from Figure 2 that the active controller can reduce the roll angle significantly compared to the passive suspension under the same lateral acceleration.

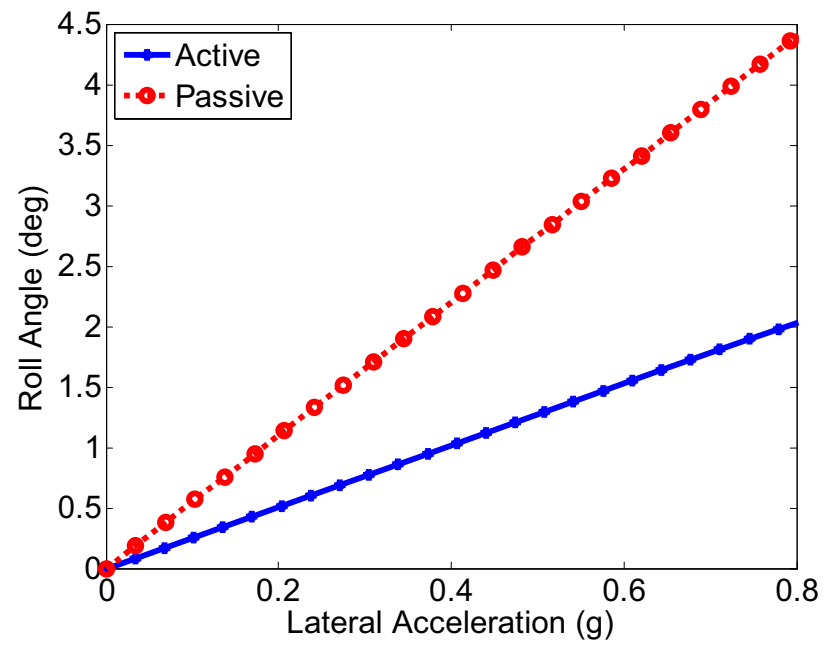

Figure 2: Steady state performance at $90 \mathrm{~km} / \mathrm{h}$

Now the steady state performance is checked when the vehicle forward speed is varied. Figure 3 shows the plot of roll angle vs forward speed under the same steer input angle. It can be seen from Figure 3 that in spite of the variation of the vehicle forward speed, the designed controller can always stabilize the system and keep the roll angle at lower values compared to the passive system. It can be seen from Figure 3 that the designed controller is robust despite the uncertainties in forward speed.
In order to show the time domain performance of the closed-loop system and the effect of time delay on the performance, one kind of maneuver, i.e. J-turn maneuver, is applied. In the J-turn maneuver, the steer input angle is given as

$\delta=\left[\begin{array}{cc}h_{0} & t<t_{0} \\ h+\left(h_{1}-h_{0}\right)\left(\frac{t-t_{0}}{t_{1}-t_{0}}\right)^{2}\left(3-2\left(\frac{t-t_{0}}{t_{1}-t_{0}}\right)\right) & t_{0}<t<t_{1} \\ h_{1} & t>t_{1}\end{array}\right.$

with $h=0 \mathrm{deg}, h_{1}=3.5 \mathrm{deg}, t_{0}=2 \mathrm{~s}, t_{1}=t_{0}+0.2 \mathrm{~s}$, which is shown in Figure 4.

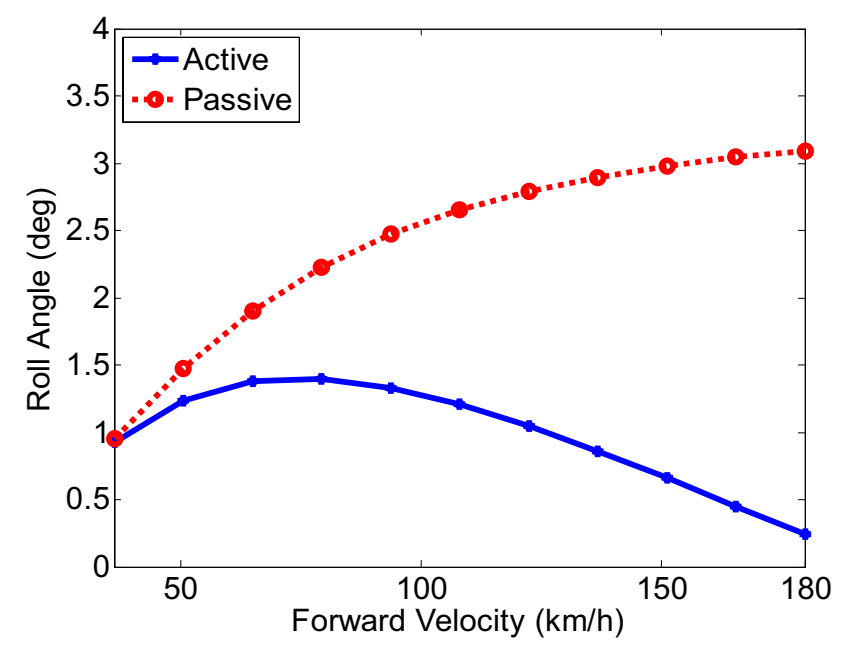

Figure 3: Steady state performance at different vehicle speed

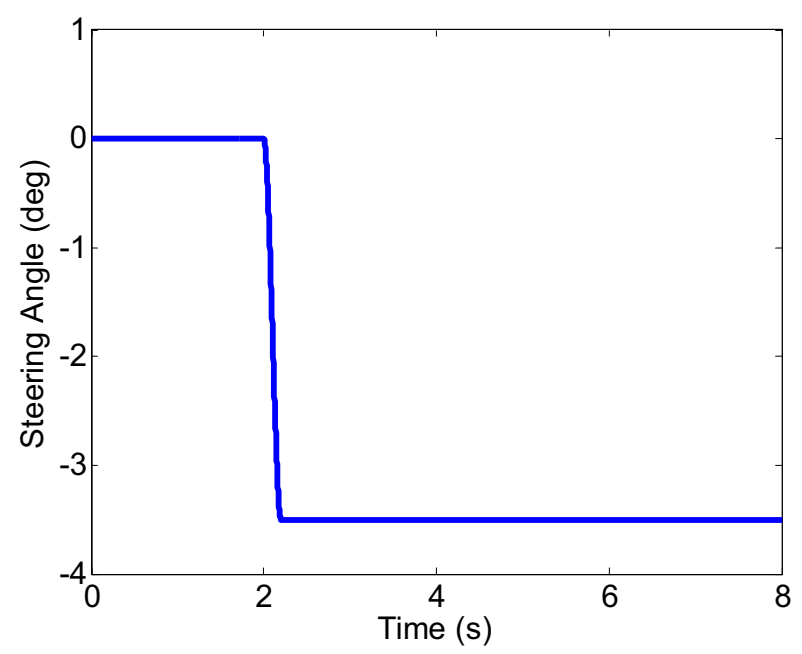

Figure 4: Steering angle during J-turn maneuver

When there is a time delay on input, i.e., $\tau=20 \mathrm{~ms}$, and forward speed is $72 \mathrm{~km} / \mathrm{h}$, the roll angle responses of the passive system and the active system are compared in Figure 5. It can be seen from Figure 5 that better response is obtained for the active case which can 
stabilize the closed-loop with no obvious degradation of performance even when $\tau=20 \mathrm{~ms}$.

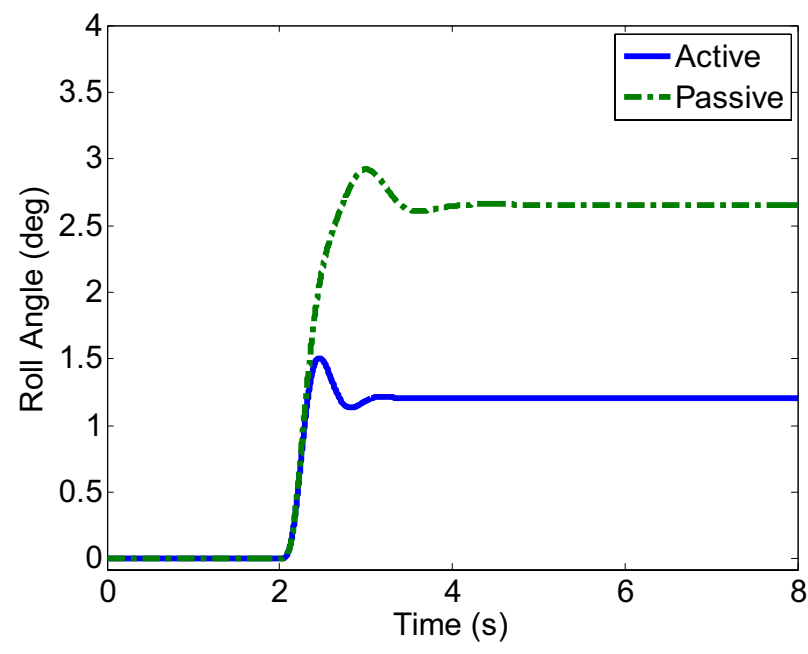

Figure 5: J-turn maneuver response when $\tau=20 \mathrm{~ms}$

To further validate the effectiveness of the designed controller in dealing with the time delay problem, the effect of time delay on the response of the roll angle is studied by calculating the steady state roll angle ratio $\phi(\tau) / \phi_{0}$ vs the time-delay $\tau$, where $\phi(\tau)$ denotes the steady state roll angle of the closed-loop system, and $\phi_{0}$ is the steady state roll angle of the open-loop system. Figure 6 shows the plot of the ratio $\phi(\tau) / \phi_{0}$ as a function of the time-delay $\tau$. It is observed from Figure 6 that the closed-loop performance is better than the open-loop system performance and there is no significant degradation in the performance of the control system up to the obtained maximal time-delay, i.e., 25 $\mathrm{ms}$. As the time-delay exceeds about $80 \mathrm{~ms}$, the degradation of the control performance increases. On the other hand, from Figure 6, we can observe the conservativeness of the presented approach. The designed maximal time-delay is $25 \mathrm{~ms}$ for the controller, however, the performance of the closed-loop can be maintained until the time-delay is about $80 \mathrm{~ms}$.

\section{CONCLUSION}

This paper presents a new controller design approach for vehicle roll control when the vehicle model is varied with forward speed and when the actuator has time delay. Based on a 3DOF roll-yaw model, the delaydependent controller is designed. It can guarantee vehicle roll performance in spite of variation in forward speed and the existence of actuator time delay. The inclusion of time delay in the control system provides a more realistic model for the applied actuator. By employing the matrix inequality formulation and genetic algorithm, the required state feedback controller can be obtained by solving a finite number of linear matrix inequalities. A simulation example is used to demonstrate that the designed controllers can effectively perform the attenuation objective even with actuator time delay and parameter uncertainties within reasonable magnitudes.

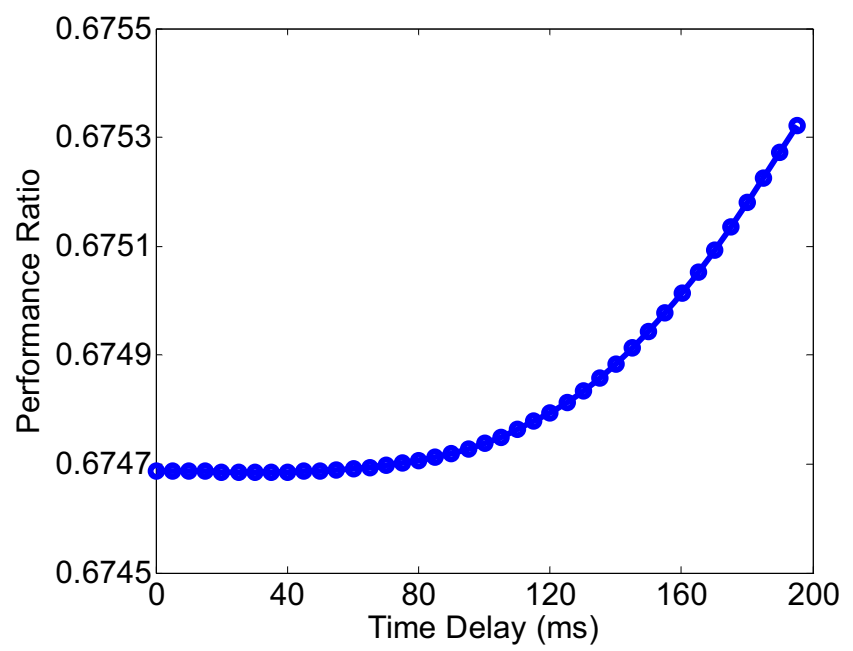

Figure 6: Roll angle ratio $\phi(\tau) / \phi_{0}$ v.s. time-delay $\tau$

\section{ACKNOWLEDGMENTS}

The financial support of this work by the Australian Research Council (DP0560077) is gratefully acknowledged. The authors would like to express their thanks to the reviewers for all the invaluable comments and suggestions leading to considerable improvements in the presentation of this paper. Thanks are also given to Mr Wade Smith, Faculty of Engineering, University of Technology, Sydney, for his help in improving the English used in the paper.

\section{REFERENCES}

1. B. C. Chen and H. Peng. Differential-braking-based rollover prevention for sport utility vehicles with human-in-the-loop evaluations. Vehicle System Dynamics, 36:359-389, 2001.

2. D. J. M. Sampson and D. Cebon. Active roll control of single unit heavy road vehicles. Vehicle System Dynamics, 40:229-270, 2003.

3. A. J. P. Miege and D. Cebon. Active roll control of an experimental articulated vehicle. Proc. Instn. Mech. Engrs. Part D, 219:791-806, 2005.

4. A. J. P. Miege and D. Cebon. Optimal roll control of an articulated vehicle: theory and model validation. Vehicle System Dynamics, 43(12):867-893, 2005.

5. H. J. Kim and Y. P. Park. Investigation of robust roll motion control considering varying speed and actuator dynamics. Mechatronics, 14:35-54, 2004.

6. P. Gaspar, I. Szaszi, and J. Bokor. Reconfigurable control structure to prevent the rollover of heavy vehicles. Control Engineering Practice, 13:699-711, 2005.

7. R. M., Palhares et al. Delay-dependent robust $H_{\infty}$ control of uncertain linear systems with lumped 
delays. IEE-Proc.-Control Theory \& Applications, 152:27-33, 2005.

8. H. Du, J. Lam, and K. Y. Sze. Non-fragile output feedback $\mathrm{H}_{\infty}$ vehicle suspension control using genetic algorithm. Engineering Applications of Artificial Intelligence, 667-680, 2003.

\section{CONTACT}

Haiping Du

\section{hdu@eng.uts.edu.au}

Mechatronics and Intelligent Systems, Faculty of Engineering, University of Technology, Sydney, P.O. Box 123, Broadway, NSW 2007, Australia.

\section{APPENDIX}

Detailed descriptions of entries in equation (1):

$$
\begin{aligned}
& \beta=\frac{v}{u_{0}}, M=M_{s}+M_{u}, Y_{\beta}=-\left(C_{\alpha f}+C_{\alpha r}\right), \\
& Y_{r}=\frac{b C_{\alpha r}-a C_{\alpha f}}{u_{0}}, Y_{\phi}=C_{\alpha r} \frac{\partial \delta_{r}}{\partial \phi}+C_{\gamma f} \frac{\partial \gamma f}{\partial \phi}, \\
& Y_{\delta}=C_{\alpha f}, N_{\beta}=b C_{\alpha r}-a C_{\alpha f}, N_{r}=-\frac{a^{2} C_{\alpha f}+b^{2} C_{\alpha r}}{u_{0}} \\
& N_{\phi}=a C_{\gamma f} \frac{\partial \gamma_{f}}{\partial \phi}-b C_{\alpha r} \frac{\partial \delta_{r}}{\partial \phi}, N_{\delta}=a C_{\alpha f}, \\
& L_{\phi}=M_{s} g h-K_{R}, L_{p}=-c_{R}, \\
& I_{x}=\left(I_{x x}\right)_{s}+M_{s} h^{2}-2 \Theta_{R}\left(I_{x z}\right)_{s}+\Theta_{R}^{2}\left(I_{z z}\right)_{s}, \\
& I_{x z}=M_{s} h c-\left(I_{x z}\right)_{s}+\Theta_{R}\left(I_{z z}\right)_{s}, \\
& I_{z}=\left(I_{z z}\right)_{s}+\left(I_{z z}\right)_{u}+M_{s} c^{2}+M_{u} e^{2} .
\end{aligned}
$$

\begin{tabular}{|c|c|c|}
\hline Name & Explanation & Value \\
\hline$M_{s}$ & Rolling sprung mass & $1663 \mathrm{~kg}$ \\
\hline$M_{u}$ & Non-rolling unsprung mass & $325 \mathrm{~kg}$ \\
\hline$\Theta_{R}$ & $\begin{array}{l}\text { Inclination angle of the roll } \\
\text { axis point down }\end{array}$ & $0.0873 \mathrm{rad}$ \\
\hline$a$ & $\begin{array}{l}\text { Distance from the vehicle } \\
\text { CG to the front axle }\end{array}$ & $1.147 \mathrm{~m}$ \\
\hline$b$ & $\begin{array}{l}\text { Distance from the vehicle } \\
\text { CG to the rear axle }\end{array}$ & $1.431 \mathrm{~m}$ \\
\hline$c$ & $\begin{array}{l}\text { Distance from the CG of } \\
M_{s} \text { to the vehicle CG }\end{array}$ & $0.421 \mathrm{~m}$ \\
\hline$e$ & $\begin{array}{l}\text { Distance from the CG of } \\
M_{u} \text { to the vehicle CG }\end{array}$ & $2.157 \mathrm{~m}$ \\
\hline$g$ & Gravity & $9.81 \mathrm{~m} / \mathrm{s}^{2}$ \\
\hline$h$ & $\begin{array}{l}\text { Distance from the CG of } \\
M_{s} \text { to the roll axis }\end{array}$ & $0.306 \mathrm{~m}$ \\
\hline$C_{\alpha f}$ & Front tire cornering stiffness & $59496 \mathrm{~N} / \mathrm{rad}$ \\
\hline$C_{\alpha r}$ & Rear tire cornering stiffness & $109400 \mathrm{~N} / \mathrm{rad}$ \\
\hline$\frac{\partial \delta_{r}}{\partial \phi}$ & $\begin{array}{l}\text { Partial derivative of the roll } \\
\text { induced steer at the rear } \\
\text { axle }\end{array}$ & 0.07 \\
\hline$\frac{\partial \gamma_{f}}{\partial \phi}$ & $\begin{array}{l}\text { Partial derivative of the } \\
\text { camber thrust at the front } \\
\text { axle }\end{array}$ & 0.8 \\
\hline$C_{\gamma f}$ & $\begin{array}{l}\text { Camber thrust coefficient at } \\
\text { the front axle }\end{array}$ & $2039 \mathrm{~N} / \mathrm{rad}$ \\
\hline$K_{R}$ & Roll stiffness & $\begin{array}{l}56957 \mathrm{Nm} / \mathrm{ra} \\
\mathrm{d}\end{array}$ \\
\hline$c_{R}$ & Roll damping coefficient & $3496 \mathrm{Nms} / \mathrm{rad}$ \\
\hline$\left(I_{x x}\right)_{s}$ & $\begin{array}{l}\text { Moment of inertia about the } \\
x \text {-axis of the rolling sprung } \\
\text { mass }\end{array}$ & $602.8 \mathrm{kgm}^{2}$ \\
\hline$\left(I_{x z}\right)_{s}$ & $\begin{array}{l}\text { Product of inertia about the } \\
x-z \text { axes of the rolling sprung } \\
\text { mass }\end{array}$ & $90.0 \mathrm{kgm}^{2}$ \\
\hline$\left(I_{z z}\right)_{s}$ & $\begin{array}{l}\text { Moment of inertia about the } \\
\text { z-axis of the rolling sprung } \\
\text { mass }\end{array}$ & $2163.7 \mathrm{kgm}^{2}$ \\
\hline$\left(I_{z z}\right)_{u}$ & $\begin{array}{l}\text { Moment of inertia about the } \\
\text { z-axis of the non-rolling } \\
\text { unsprung mass }\end{array}$ & $540.0 \mathrm{kgm}^{2}$ \\
\hline
\end{tabular}

Table 1: Vehicle Parameter for a 1997 Jeep Cherokee 